\title{
ANNALS OF GEOPHYSICS: AD MAJORA
}

\author{
Fabio Florindo ${ }^{1}$, Francesca Bianco ${ }^{2}$, Paola De Michelis ${ }^{1}$, Simona Masina ${ }^{3,4}$, Giovanni Muscari ${ }^{1}$, \\ Francesco Caprara ${ }^{3}$, Anna Grazia Chiodetti ${ }^{3}$, Edoardo Del Pezzo ${ }^{2}$, Stefano Gresta ${ }^{1,5}$
}

\author{
${ }^{1}$ Istituto Nazionale di Geofisica e Vulcanologia, Via di Vigna Murata 605, 00143, Rome, Italy \\ ${ }^{2}$ Istituto Nazionale di Geofisica e Vulcanologia, Sezione “Osservatorio Vesuviano", Via Diocleziano 328, 80124, Naples, Italy \\ ${ }^{3}$ Istituto Nazionale di Geofisica e Vulcanologia, Via Donato Creti 12, 40128, Bologna, Italy \\ ${ }^{4}$ Centro Euro-Mediterraneo sui Cambiamenti Climatici, 40128, Bologna, Italy \\ ${ }^{5}$ Università di Catania, Dipartimento Scienze Biologiche, Geologiche e Ambientali, Corso Italia 57, 95129, Catania, Italy
}

Annals of Geophysics (ISSN: 1593-5213; from 2010, 2037-416X) is a bimonthly international journal, which publishes scientific papers in the field of geophysics sensu lato. It derives from Annali di Geofisica (ISSN: 0365-2556), which commenced publication in January 1948 as a quarterly periodical devoted to general geophysics, seismology, Earth magnetism, and atmospheric studies (Figure 1). The journal was published regularly for a quarter of a century until 1982 when it merged with the French journal Annales de Géophysique to become Annales Geophysicae (ISSN: 0755-0685) under the aegis of the European Geophysical Society. In 1981, this journal ceased publication of the section on solid Earth geophysics, ending the legacy of Annali di Geofisica. In 1993, the Istituto Nazionale di Geofisica (ING), founder of the journal, decided to resume publication of its own journal under the same name, Annali di Geofisica. To ensure continuity, the first volume of the new series was assigned the volume number XXXVI (following the last issue published in 1982). In 2002, with volume XLV, the name of the journal was translated into English to become Annals of Geophysics and in consequence the journal impact factor counter was restarted.

Starting in 2010, in order to improve its status and better serve the science community, Annals of Geophysics has instituted a number of editorial changes including full electronic open access, freely accessible online, the possibility to comment on and discuss papers online, and a board of editors representing Asia and the Americas as well as Europe. To celebrate this major change to the modern online format, the first volume of the new Annals of Geophysics was produced as a historical volume in which we published a set of selected papers by eminent authors who, in different ways and in different contexts, have provided us with important benchmarks in the development of geophysics [e.g., Aki et al. 2010, Båth 2010, Gutenberg and Richter 2010, Lehmann 2010]. All of these papers were published before 1977 in Annali di Geofisica, as the journal was then called.

In March 2013, sixty-five years after the journal foundation and three years after the relaunch of Annals of Geophysics as an online open-access journal (we use Open Journal Systems 2.4.1.0, which is open source journal management and publishing software developed, supported, and freely distributed by the Public Knowledge Project), which certainly contributed to its international success, we proceeded with a partial re-

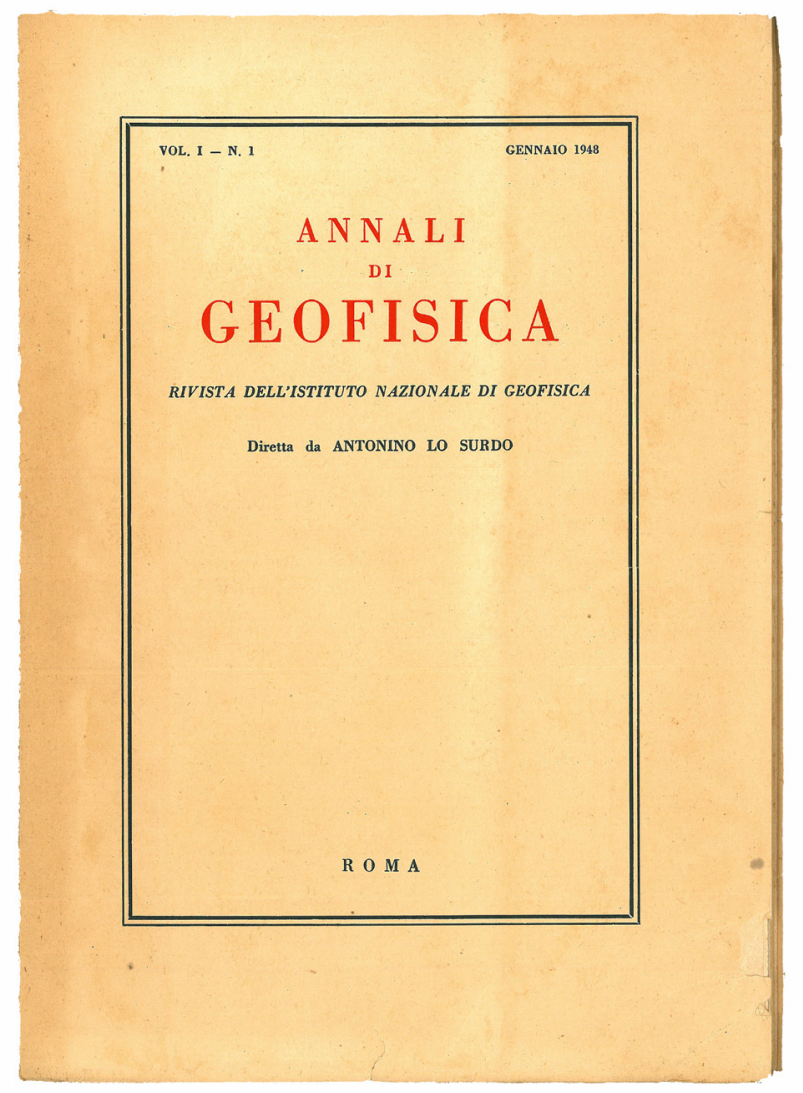

Figure 1. First issue of Annali di Geofisica, 1948. 


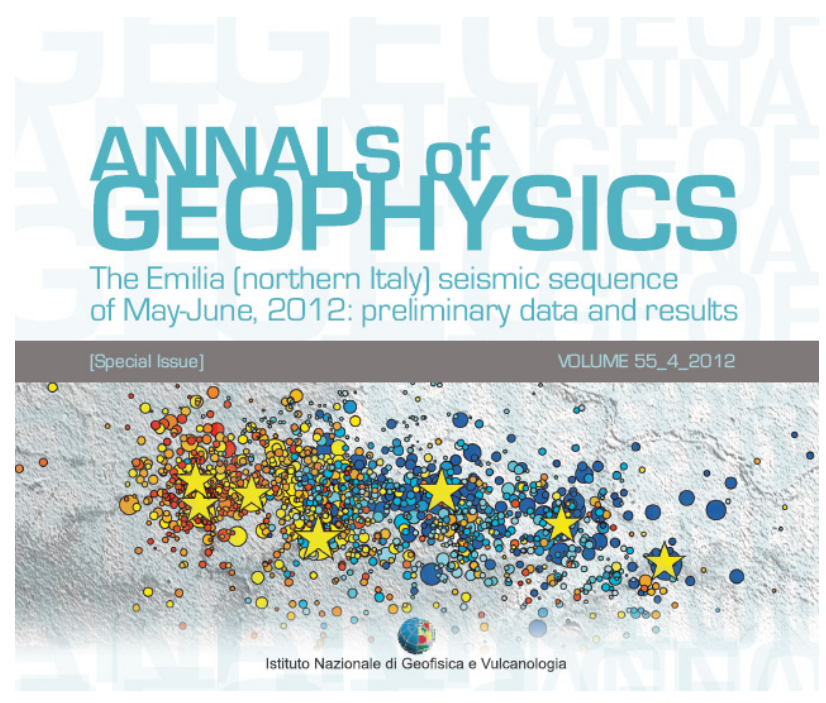

Figure 2. Cover of the Annals of Geophysics fast track issue on the Emilia (northern Italy) seismic sequence of May-June, 2012.

organization of the journal itself.

In addition to "Research Articles", Annals of Geophysics now publishes several other categories of papers including "Scientific News", "Data and Experiment Description", "Review Articles", "Letters", and "Fast Track" papers. Data and experiments descriptions is a kind of paper in which data collected during important experiments or field surveys (seismology during eruptions, aftershocks of main earthquakes having particular impact and so on) are described and put in sharing with the scientific community. The Journal itself can act as data repository. The latter two categories are among the most recent addition and allow scientists to quickly publish the preliminary results of their findings. The first issues including "Letters" and "Fast Track" papers were volume 56, 2013, on Atmospheric Composition Validation and Evolution - ACVE 2013 [e.g., Richter et al. 2013] and volume 55 (4) on The Emilia (northern Italy) seismic sequence of May-June, 2012: preliminary data and results, which was entirely dedicated to this dramatic seismic sequence [Anzidei et al. 2012] (Figure 2).

The journal is also active in providing assistance to conveners of important national and international meetings and workshops, for the publication of papers therein presented, and will publish special volumes on specific topics of broad interest to the scientific community. Recent special issues include volume 55 (3), 2012, on Geoethics and geological culture. Reflections from the Geoitalia Conference 2011 [Peppoloni and Di Capua 2012] with a collection of papers presented during the 'Geoethics' scientific session at the 8th Italian Forum of Earth Sciences that was held in September 2011 in Turin, Italy, and volume 55 (6), 2012, entitled 5th European Repeat Station (MagNetE) Workshop Rome, Italy 2011 [De Michelis and Duma 2012] which contains a subset of the scientific results presented at the 2011 MagNetE symposium. One of the next goal will be to re-organize the publication of special volumes focused onto scientific topics that are generally considered as top-arguments in the fields of geophysics and volcanology.

As part of the journal's reorganization we have appointed four section editors covering four main research areas (1. seismology, volcanology, geodesy and geochemistry; 2. geomagnetism, paleomagnetism and solar terrestrial physics; 3 . atmospheric physics and chemistry; 4. physical oceanography and climatology), 22 new associate editors, and a new editor-in-chief. Like all peer-reviewed journals, Annals of Geophysics has a board of associate editors comprising leading national and international experts on the various section topics of the journal. The associate editors provide the section editors and the editor-in-chief with advice on choosing referees, assist in reviewing papers themselves, undertake book reviews, and may contribute editorials to the journal. The section editors need to ensure both that they have the right balance of expertise available to them for the papers that the journal is receiving, and that the role of associate editor is spread across the community.

Since 2002 the number of citations has been steadily increasing (Figure 3) and in 2012 the impact factor increased to 1.138, placing Annals of Geophysics in a respectable position among the Geochemistry and Geophysics subject category of ISI Web of Science.

None of this progress would have been possible without the commitment of authors, reviewers, and previous editors, and we are grateful for all of the effort they have expended on the journal. For further information about the journal's scope, submission formats, and review and production procedures, please see http: / / www.annalsofgeophysics.eu.

We look forward to handling your paper.

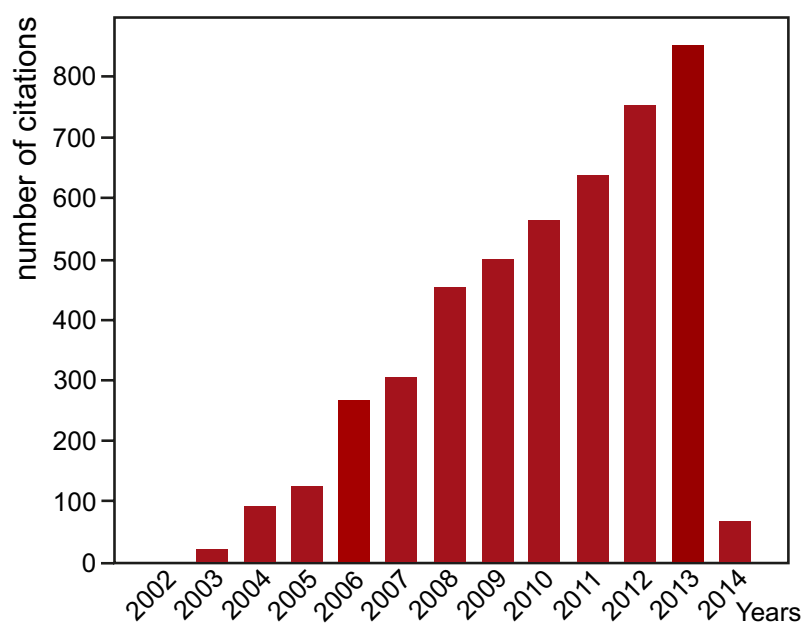

Figure 3. Number of citations per year (source: Web of Science). 


\section{References}

Aki, K., M. Bouchon, B. Chouet and S. Das (2010). Quantitative prediction of strong motion for a potential earthquake fault, Annals of Geophysics, 53 (1), 81-91; doi:10.4401/ag-4665.

Anzidei, M., A. Maramai and P. Montone (2012). Preface, Annals of Geophysics, 55 (4), 515; doi:10.4401/ ag-6232.

Båth, M. (2010). Teleseismic magnitude relations, 53 (1), 69-80; doi:10.4401/ag-4664.

De Michelis, P., and G. Duma (2012). Preface, Annals of Geophysics, 55 (6), 1051; doi:10.4401/ag-6313.

Gutenberg, B., and C.F. Richter (2010). Magnitude and energy of earthquakes, Annals of Geophysics, 53 (1), 7-12; doi:10.4401/ag-4588.

Lehman, I. (2010). On amplitudes of P near the shadow zone, Annals of Geophysics, 53 (1), 13-15; doi:10.44 $01 /$ ag-4589.

Peppoloni, S., and G. Di Capua (2012). Foreword and Acknowledgements, Annals of Geophysics, 55 (3); doi:10.4401/ag-6100.

Richter, A., M. Weber, J. P. Burrows, J.-C. Lambert and A. van Gijsel (2013). Validation strategy for satellite observations of tropospheric reactive gases, Annals of Geophysics, 56, Fast Track 1, 1-10; doi:10.4401/ ag-6335. 